
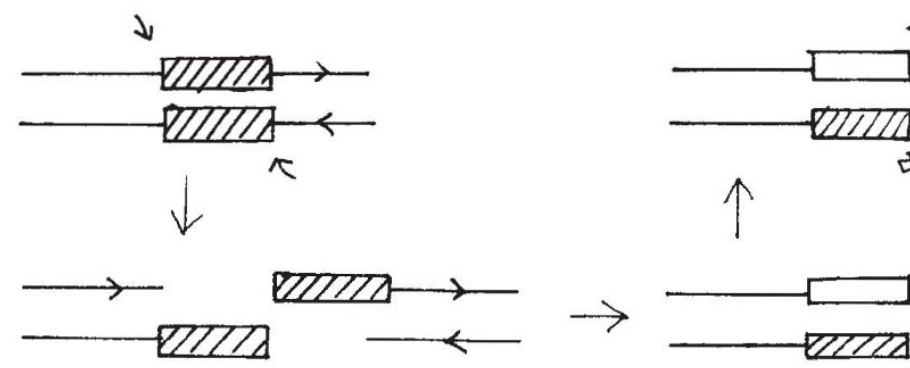

Fig. 2

interestingly other direct duplications result from transpositional events. When bacteriophage $\mathrm{Mu}$ is used to mediate transposition of a bacterial gene $X$, to a new replicon, $X$ is finally found to be bounded by directly repeated $\mathrm{Mu}$ genomes. Similarly, experiments in Falkow's laboratory in Seattle and ours in Brighton, have shown that transposition of one class of mutant transposon from replicon $A$ to replicon $\mathrm{B}$, results in the acquisition by $\mathbf{B}$ of two directly repeated copies of the transposon sandwiching replicon A.

Sequence analysis of IS 1 (N. Grindley; Ohtsubo \& Ohtsubo Proc. natn. Acad. Sci. U.S.A. 75, 615; 1978) and part of $\operatorname{Tn} 3$ (Cohen, Chou \& Casadaban) shows a number of interesting features. IS 1 has at its ends an inverted repeat of 30 bases while that of $\operatorname{Tn} 3$ is somewhat larger. Neither set of repeats is perfect and those of IS1 contain a number of promoterlike sequences as well as a sequence

\section{Hunting the W}

\section{from Peter Landshoff}

It seems that there are in nature four different types of force between particles: the strong interaction that holds protons and neutrons together inside a nucleus, the electromagnetic interaction, the weak interaction responsible for nuclear beta decay, and the very much feebler gravitational interaction. Of these, the electromagnetic force is the best understood: it is described to extremely high accuracy by quantum electrodynamics. This theory quantises Maxwell's classical electromagnetic field, so that the field is given a particlelike character. The particles, photons, transmit the electromagnetic force between charged particles.

It has been believed for many years that there are also particles that transmit the weak force. These particles are

Peter Landshoff is Reader in Mathematical Physics at the University of Cambridge.
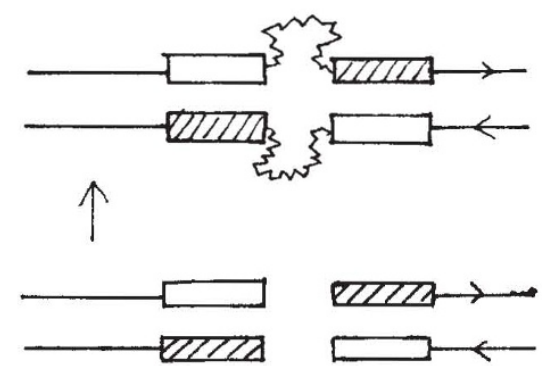

Salam and Weinberg have formulated an elegant quantum field theory which incorporates both the electromagnetic and the weak interactions. Their model is a first step in the unification of all four fundamental forces into a single relativistic quantum theory, which has long been an ambition of theoretical physicists. Predictions of the model agree well with experiment but the existence of $\mathrm{W}$ particles is crucial for its validity. In order that the Salam-Weinberg model be internally self-consistent, the mass of the $W$ particles must be greater than $37.5 \mathrm{GeV} / c^{2}$, but a value close to twice this makes the model agree best with the details of the neutrino scattering data.

There are various plans, on both sides of the Atlantic, to construct accelerators of energy high enough to produce particles of such large mass. The Super Proton Synchrotron (SPS) in the European laboratory at CERN is particularly well suited to be adapted to provide the necessary energy. At present, the SPS accelerates protons to energy $400 \mathrm{GeV}$. They are extracted from the machine and are made to scatter on fixed targets. It is planned to inject into the SPS simultaneously both protons and antiprotons; these will circulate in the accelerator ring in opposite directions and will be made to collide head-on. Each particle in the head-on collisions will have energy 270 $\mathrm{GeV}$; according to special relativity the available energy in each collision then is equal to that of a $150,000 \mathrm{GeV}$ proton colliding with a fixed target.

At such high energy many interesting things are expected to occur as a result of the proton-antiproton interaction, among them the production of $\mathrm{W}$ particles. If the SPS collider performs as well as is hoped, there will be at least 10,000 interactions per second, and each interaction is expected to produce about 25 particles, mostly pions. There are indications from cosmic ray studies that in some interaction events the multiplicity will be considerably higher than this. In order to cope with the full complexity of the events, a sophisticated particle detector will be needed. A collaboration of more than 50 physicists, from Aachen, Annecy, Birmingham, California, CERN, Collège de France, Queen Mary College, Rutherford Laboratory and Saclay is proposing to build a huge detector, weighing 1,500 tons. As the SPS is underground the detector will have to be assembled $25 \mathrm{~m}$ below ground level.

It is estimated that one proton-antiproton interaction in every 10 million will produce a $\mathrm{W}$ particle. It may not be straightforward to recognise these events but, nevertheless, the race to find the $\mathrm{W}$ is on, and Europe hopes to win it. 